\title{
Preparação psicológica para admissão hospitalar de crianças: uma breve revisão
}

\author{
Fernanda Nascimento Pereira Doca \\ Áderson Luiz Costa Junior \\ Universidade de Brasília, Brasília-DF, Brasil
}

Resumo: A preparação psicológica constitui uma ampla modalidade de intervenção, que inclui diferentes procedimentos e técnicas, recomendadas por pesquisadores em psicologia da saúde e da psicologia pediátrica para uso com pacientes e familiares expostos a condições potencialmente estressantes. Entre os benefícios apontados consta a redução de efeitos aversivos associados à experiência de hospitalização, o aumento dos índices de adesão ao tratamento e o desenvolvimento de estratégias mais eficientes de enfrentamento. No entanto, observa-se que a literatura ainda não dispõe de uma sistematização metodológica suficiente que permita apontar relações funcionais precisas entre intervenção e efeitos. Este trabalho tem por objetivo efetuar uma revisão bibliográfica sobre o tema da preparação psicológica para hospitalização da criança, destacando princípios metodológicos que envolvem o planejamento e a execução da intervenção e propor, também, um modelo de programa de preparação psicológica para admissão hospitalar infantil.

Palavras-chave: Preparação psicológica. Psicologia pediátrica. Hospitalização infantil. Admissão hospitalar.

\section{Psychological preparation for pediatric hospital admission: a short review}

\begin{abstract}
The psychological preparation for hospital admission in children constitutes an intervention modality, that includes different procedures and techniques, recommended by professionals of health and pediatric psychology to be used with patients and families exposed to potentially stressing conditions. Some benfits may be highlighted as reduction of the stress effect associated to the hospitalization experience, increased adherence to the treatment and the development of better coping strategies to face the treatment. However, the literature still doesn't have a systematic methodology that would help to understand the functional relationship between intervention and effects. This study contains a bibliographical revision about the psychological preparation for hospitalization, points out some methodological principles involved in the intervention planning and execution, and proposes a psychological preparation model program for pediatric hospital admissions.
\end{abstract}

Keywords: Psychological preparation. Pediatric Psychology. Pediatric hospitalization. Hospital admission.

\section{Preparación psicológica para hospitalización infantil: un breve repaso}

Resumen: La preparación psicológica constituye una amplia modalidad de intervención, que incluye distintos procedimientos y técnicas, recomendadas por investigadores en psicología de la salud y de la psicología pediátrica para uso con pacientes y familiares expuestos a condiciones potenciales de estrés. Entre los beneficios señalados están la reducción de los efectos de aversión asociados a la experiencia de hospitalización, el aumento de los índices de adhesión al tratamiento y el desarrollo de estrategias más eficientes de afrontamiento. Sin embargo, se nota que la literatura todavía no dispone de una sistematización metodológica suficiente que permita apuntar relaciones funcionales bien delimitadas entre intervención y efectos. Este estudio se propone a realizar un repaso bibliográfico acerca del tema de preparación psicológica para la hospitalización de niños, subrayando principios teóricos y metodológicos que incluyen el planeamiento y la realización de la intervención y propone también un modelo de programa de preparación psicológica para admisión hospitalaria infantil.

Palavras clave: Preparación psicológica. Psicología pediátrica. Hospitalización infantil. Admisión hospitalar. 


\section{Introdução}

Adoecer, um evento não esperado e, na maior parte das vezes, não desejado, implica na exposição do indivíduo a vulnerabilidades de natureza física, social e psicológica, condicionadas por uma ampla variedade de fatores, tais como: a gravidade da patologia, os efeitos colaterais desagradáveis de determinados tratamentos, o estigma social vinculado à doença e as restrições ambientais impostas pelo tratamento (Barros, 1999; Zannon, 1981). O adoecimento afeta a rede de apoio do indivíduo e, no caso da criança, afeta, principalmente, seus cuidadores primários (Barros, 1999; Guimarães, 1988; Quiles \& Carrillo, 2000; Scott \& Arslanian-Engoren, 2005).

Do ponto de vista psicológico, a experiência de adoecimento envolve inúmeras perdas transitórias e/ou permanentes e quando se faz necessária a internação hospitalar, o caráter potencial estressante do adoecimento é freqüentemente aumentado (Barros, 1999; Oliveira, 1997).

Destaca-se que o modo como se processa a admissão hospitalar pode constituir uma experiência adversa de desenvolvimento, condicionando o modo como paciente e acompanhante vão enfrentar o período de confinamento hospitalar e os múltiplos eventos do tratamento. Contudo, intervenções psicológicas podem reduzir o impacto desta experiência tanto para a criança e quanto para seu acompanhante.

Para tanto, a literatura em psicologia da saúde preconiza a necessidade e a relevância de uma preparação psicológica sistematizada para a internação hospitalar de crianças (Roberts, 2003). Pesquisas em psicologia pediátrica revelam resultados positivos de procedimentos de preparação psicológica, especialmente no que se refere ao aumento dos índices de adesão ao tratamento, redução de ansiedade antecipatória e desenvolvimento de estratégias de enfrentamento mais eficientes (Anderson \& Collier, 1999; Burke, Harrison, Kauffmann \& Wong, 2001; Corr, 1991; Martins, Ribeiro, Borba \& Silva, 2001; Quiles \& Carrillo, 2000; Soares \& Bomtempo, 2004).

No entanto, deve-se destacar que a maior parte dos estudos investiga efeitos da preparação psicológica para eventos específicos da internação pediátrica e não para o processo de internação como um todo. Ainda assim, as pesquisas não apontam resultados consistentes ou suficientemente conclusivos que permitam especificar metodologias precisas ou modelos de operacionalização que garantam a obtenção generalizada de efeitos positivos da intervenção psicológica sobre o comportamento de crianças expostas a eventos específicos da internação hospitalar, tais como a submissão a procedimentos médicos invasivos (Araujo \& Tubino, 1996; Betz, 2006; Bragado Álvarez \& Fernandéz, 1997; Costa Junior, 2004). Parte desta falta de sistematização metodológica pode ser atribuída à complexidade clínica, à multiplicidade de variáveis psicossociais relacionadas ao contexto de tratamento de saúde e às diversas abordagens teórico-filosóficas que subsidiam as intervenções psicológicas.

No Brasil, a situação não é diferente. Além das diversas variáveis envolvidas e do reduzido número de estudos que investigam sistematicamente os efeitos da preparação psicológica para internação hospitalar de crianças (Costa Junior, 2004), observase que parcela significativa dos psicólogos brasileiros tem recebido formação acadêmica deficitária, especialmente no que se refere à metodologia de pesquisa científica em psicologia da saúde, psicologia pediátrica, psico-oncologia, entre outras. Verifica-se, em congressos nacionais e eventos científicos destas áreas, grande quantidade de relatos de experiência profissional sem qualquer sistematização ou controle sobre as intervenções e carência de medidas precisas de seus efeitos (Costa Junior, 2001).

Este trabalho teve por objetivo efetuar uma revisão da literatura sobre o tema da preparação psicológica de crianças para hospitalização, destacando princípios metodológicos que envolvem as intervenções. Em seguida, propõe-se um modelo de programa de preparação para a admissão hospitalar que está em fase de implantação no Centro de Pediatria Clínica do Hospital Universitário de Brasília (HUB).

As referências utilizadas nesta breve revisão foram divididas em dois grandes grupos. O primeiro trata de trabalhos publicados nas três últimas décadas, especificamente de 1981 a 2006, referentes à contextualização da hospitalização infantil. O segundo grupo se refere à intervenção de preparação psico- 
lógica, subsidiada principalmente por artigos publicados no período de 2000 a 2006, em bases de dados indexadas no Portal Capes de Periódicos.

\section{A internação}

A experiência de adoecimento exige que o indivíduo tenha de lidar com demandas que, freqüentemente, ultrapassam sua capacidade de adaptação cotidiana. Na proposta biocomportamental do desenvolvimento humano, adoecimento e internação são eventos de transição ecológica que alteram o sistema de comportamentos do indivíduo com seus ambientes próximos (Bronfenbrenner, 1979). Desta forma, alteram-se os papéis sociais (familiar, ocupacional, entre outros) e as relações interpessoais do indivíduo. Com a internação hospitalar, o indivíduo também fica exposto a diversas experiências de perda, temporárias ou não, que envolvem: (a) seu bem-estar físico e psicológico; (b) seu ambiente natural e pertences pessoais; (c) a tomada de decisões em relação à sua rotina diária; (d) o controle sobre si mesmo e sobre o ambiente; e (e) a relação com as pessoas de sua rede social (Barros, 1999; Junqueira, 2003; Oliveira, 1993, 1997). O paciente, no hospital, habita um local estranho; utiliza uma vestimenta padronizada e fornecida pela instituição, pouco funcional, de privacidade reduzida e nem sempre ajustada ao seu corpo físico; segue horários de alimentação, sono e banho, definidos institucionalmente, bem como condutas impostas pela equipe de saúde (Barros, 1999; Guimarães, 1988; Oliveira, 1997).

Assim, a internação hospitalar exige, do paciente, adaptações comportamentais em tempo nem sempre suficiente para os ajustes emocionais e cognitivos a que os esquemas e crenças subjacentes podem proceder (Beck, 1976). Como resultado, conforme descrevem Lazarus e Folkman (1984), a exposição a ambientes cujas exigências de adaptação ultrapassam os limites suportáveis pelo indivíduo constitui um preditor de estresse e de outras respostas desadaptativas, que, na internação de crianças, podem ser observadas por meio de comportamentos de rebaixamento do humor, protestos, retraimento social e desmotivação (Roberts, 2003).

Destaca-se que, na prática, quando o paciente internado é uma criança, as perdas e os fatores de estresse afetam, mais diretamente, seus familiares. Considerando-se que na cultura ocidental, a criança é percebida como um indivíduo ingênuo, frágil, rico em possibilidades e longevidade, na internação infantil freqüentemente se observa relatos verbais dos adultos indicando sentimentos de raiva, piedade e não aceitação da situação. Estes, por sua vez, podem afetar o estado emocional da criança e seu repertório de comportamentos diante da doença e do tratamento, especialmente quando o adulto é a mãe, o pai ou pessoa de referência pessoal da criança (Costa Junior, 2004).

Pesquisas em psicologia pediátrica têm apontado o comportamento dos pais como principal modelo condicionador do comportamento da criança, especialmente quando se espera colaboração da criança para a execução de exames e procedimentos terapêuticos (Blount, Landolf-Fritsche, Powers \& Sturges, 1991; Blount, Piira, Cohen \& Cheng, 2006; Blount e cols., 1997; Crawford, 2002). Vários estudos demonstram que os comportamentos dos pais influenciam intensamente as reações emocionais da criança e sua percepção da experiência de hospitalização (Barros, 1999). Conforme observou Costa Junior (2004), comportamentos de pais e acompanhantes, caracterizados como de baixa tolerância e concorrentes com os eventos do tratamento, podem configurar uma condição de ansiedade, generalizando-se para a criança e dificultando, ou mesmo impedindo, a atuação dos profissionais de saúde.

Os resultados destes estudos, portanto, indicam que os pais podem ser elementos-chave no processo de adaptação e enfrentamento da hospitalização infantil, ao oferecerem modelos funcionais e adequados, ao estimularem o desenvolvimento destas habilidades em seus filhos e ao oferecer-lhes apoio emocional incondicional. No entanto, para que isso ocorra, pode ser necessário disponibilizar treinamento e suporte emocional especializado aos pais e acompanhantes (Costa Junior, 2004).

Mas, quais são as repercussões adversas da hospitalização para a criança e para seus pais? De acordo com estudos em psicologia pediátrica, as repercussões adversas da hospitalização à criança incluem: (a) regressões de comportamento; (b) 
alterações do humor e de comportamento social; (c) expressão de medos; (d) transtornos do sono; (e) transtornos alimentares; e (f) agressividade (Barros, 1999; Crepaldi, Rabuske \& Gabarra, 2006; Quiles \& Carrillo, 2000). É importante ressaltar que, para alguns autores, estas alterações podem ocorrer durante a internação e persistir por períodos posteriores à alta ou até mesmo perdurar por toda a vida, visto alterarem o curso de desenvolvimento normal da criança (Barros, 1999; Forinder, 2004; Quiles \& Carrillo, 2000).

Para os pais, as repercussões adversas da hospitalização infantil estão mais relacionadas ao período de internação e/ou tratamento, contudo, também podem estender-se por prazos mais longos, especialmente nos casos em que seqüelas incapacitantes, danos irreversíveis ou óbito são verificados (Chiattone, 2001). As repercussões mais comuns aos pais incluem: (a) manifestações de ansiedade; (b) depressão; (c) confusão mental; (d) alterações do ciclo sono-vigília; (e) transtornos alimentares; e (f) pensamentos repetitivos e intrusivos (Quiles \& Carrillo, 2000).

Tendo em vista estas implicações adversas ao processo de desenvolvimento humano, tanto da criança quanto de seus pais, observa-se que a psicologia pediátrica tem se preocupado com o desenvolvimento de estratégias de intervenção psicossocial, tais como a preparação psicológica, que minimizem os efeitos adversos da hospitalização infantil e dos eventos do tratamento.

\section{A preparação psicológica}

A preparação psicológica é caracterizada por Quiles e Carrillo (2000) como um conjunto de procedimentos técnicos que tem por objetivo geral aliviar as reações emocionais advindas da situação de internação hospitalar e facilitar a adaptação comportamental a este contexto. Em função do momento de ocorrência, a preparação pode acontecer em três fases distintas: (a) antes da hospitalização; (b) no momento da admissão hospitalar; ou (c) antes de exames e procedimentos médicos invasivos, tais como punções e cirurgias.

Os programas de preparação psicológica executados antes da internação constituem ações preventivas, de cunho educativo, dirigidos a crianças sem experiência de hospitalização, e podem ser desenvolvidos tanto na comunidade, quanto em ambulatório. Na comunidade, o mais comum são os programas escolares nos quais as crianças assistem palestras e filmes, têm acesso a manuais (guidelines), discutem conteúdos de saúde com especialistas e desenvolvem atividades didáticas que envolvem temáticas de adoecimento, tratamento médico e hospitalização. Nos programas desenvolvidos em ambulatório, observam-se ações mais específicas, dirigidas ao agendamento e realização de visitas técnicas de uma criança, ou grupos de crianças, ligadas, ou não, a algum programa escolar, ou que apresentem algum risco particular de internação. Os resultados precisos dessas intervenções ainda não são suficientemente conhecidos devido à escassez de pesquisas longitudinais que avaliem o desempenho, ao longo da internação, de uma criança preparada a partir de uma destas metodologias (Barros, 1999).

Os programas de preparação desenvolvidos no momento da admissão hospitalar mantêm o caráter preventivo dos anteriores e envolvem o fornecimento de informações sobre normas e rotinas da unidade, procedimentos que serão executados e treino em habilidades de enfrentamento. Em ordem decrescente, as formas mais comuns de repasse dessas informações são: (a) oral, durante os atendimentos ao paciente; (b) impressa, por meio de folhetos e cartilhas; e (c) audiovisual, por meio da projeção de slides e filmes de curta duração (Barros, 1999; Crawford, 2002; Scott \& Arslanian-Engoren, 2005).

Por fim, os programas de preparação psicológica em período imediatamente antecedente à execução de procedimentos médicos invasivos são os mais pesquisados e envolvem a maior variabilidade de metodologias. Destacam-se três modalidades de preparação em função dos objetivos: (a) fornecimento de informações orais e/ou escritas, familiarizando o paciente com a situação a ser enfrentada; (b) distração, por meio de jogos, brincadeiras e atividades concorrentes com o procedimento médico a ser realizado, de modo a desviar a atenção do paciente; e (c) simulação da situação real por meio de jogos, audiovisuais e desempenho de papéis, facilitando a adaptação comportamental da criança à situação 
(Cavender, Goff, Hollon \& Guzzetta, 2004; Franck \& Jones, 2003; Martins \& cols., 2001; World Health Organization, 1993).

Considerando-se esses três momentos de preparação psicológica, bem como as características de cada um deles, podemos questionar os motivos da maior concentração de programas e pesquisas no período que antecede a exposição a procedimentos médicos invasivos.

A resposta parece estar relacionada a vários fatores. Um deles se refere à vigência do modelo biomédico de saúde, o qual se caracteriza: (a) pelo foco nas intervenções curativas e não nas preventivas; (b) na doença e não no indivíduo que a manifesta; (c) pelo incentivo ao alto nível de especialidade tanto das técnicas quanto dos profissionais que a executam; e (d) pela visão dualista, que preconiza mente e corpo como entidades separadas, não reconhecendo o processo saúde-doença como um objeto único, uma continuidade (Guimarães, 1999; Ogden, 1999; Reis, 1998; Roberts, 2003).

Outro fator se refere ao potencial aversivo e estressor da submissão a determinados procedimentos invasivos (Blount \& cols., 2006; Ramsay, 2000; Willemsen, Chowdhury \& Briscall, 2002), o que desperta grande interesse entre os pesquisadores, que têm desenvolvido e testado procedimentos voltados à redução da freqüência de comportamentos indicadores de sofrimento do paciente ou comportamentos concorrentes aos procedimentos médicos e odontológicos invasivos (Costa Junior, 2002, 2004; Soares \& Bomtempo, 2004).

Segundo Blount e cols. (2006), os programas de preparação psicológica devem priorizar a redução da percepção de dor durante a exposição a procedimentos médicos-hospitalares e não prevenir a sua ocorrência, ou ensinar a lidar com ela, a partir de intervenções ao início da internação. Estes autores destacam os efeitos positivos de intervenções cognitivo-comportamentais como redutores de queixas de dor, enfocando a efetividade da distração (por meio do redirecionamento da atenção da criança para estímulos não relacionados ao tratamento) e o treino dos pais como agentes estimuladores do comportamento de colaboração de seus filhos.

Neste sentido, pode-se apontar que apesar dos esforços da psicologia da saúde pelo desenvolvimento de propostas promocionais que garantam condições cada vez mais eficientes de saúde, grande parte dos pesquisadores em psicologia pediátrica dedica-se a ações de apenas cunho curativo. Tais observações parecem refletir as conseqüências de sistemas de saúde não planejados para ações preventivas primárias, de identificação de riscos potenciais, mas voltados ao atendimento de ações terciárias e quaternárias de saúde, com alta tecnologia e alto custo de resposta.

Ramsay (2000), por exemplo, destaca que os programas de preparação psicológica devem focalizar a experiência de dor entre adultos e crianças, especialmente na condição de pós-operatório. A abordagem deve ser individual, dado o envolvimento de grande carga de fatores emocionais e perceptivos, além da necessidade de habilidades para comunicarse e compreender conceitos e contextos específicos. $\mathrm{O}$ autor propõe uma abordagem multimodal, com o uso de diferentes substâncias farmacológicas e técnicas comportamentais que possam trazer melhores benefícios e reduzir os efeitos colaterais aversivos dos procedimentos cirúrgicos.

Cavender e cols. (2004) efetuaram um estudo com uma amostra de 43 crianças submetidas à punção venosa; o grupo de controle recebeu suporte e cuidados padronizados durante a punção venosa e o grupo experimental teve o acréscimo do treinamento de seus pais, que foram orientados a manterem as crianças concentradas e engajadas em atividades de distração. Os resultados indicaram redução significativa de medo e estresse entre as crianças do grupo experimental.

Em estudo que investigava fobia de agulha em crianças, Willemsen e cols. (2002) disponibilizaram várias opções de tratamento, incluindo anestesia local, apoio educacional com explicação e/ou orientação, relaxamento, distração, dessensibilização e reestruturação cognitiva, destacando que a escolha da técnica e sua aplicação estão relacionadas funcionalmente às necessidades psicossociais da criança, que devem ser identificadas pelo profissional de saúde.

Observa-se que a literatura destaca a importância do treinamento da equipe de saúde para a adoção de programas de preparação para procedimentos médicos invasivos. Koehn (2006), por 
exemplo, enfatiza a necessidade do treinamento da equipe médica para o desenvolvimento de habilidades interpessoais, de modo a serem capacitados para o trabalho interdisciplinar e para desenvolver boas relações com os pacientes e seus familiares. $\mathrm{O}$ autor propõe um modelo curricular para o ensino de medicina dividido em cinco domínios, um para cada habilidade a ser treinada entre os futuros médicos: clínico-analítico, emocional, criativa, comunicativa e funcional.

Por sua vez, Wade (1999) destaca o treinamento da equipe de enfermagem, especialmente o desenvolvimento de habilidades psicossociais, por ser esta a equipe que lida diretamente com o paciente no dia-a-dia da internação. Para o treinamento, Wade propõe o uso de estudos de caso como estratégia de ensino, visto que estimulam o pensamento crítico, facilitam a compreensão da informação e proporcionam um entendimento da natureza holística e da complexidade de pacientes e famílias de alto risco.

Contudo, alguns estudos apontam que o treinamento de equipes nem sempre é suficiente. Como os pais exercem influência significativa no comportamento dos filhos, também requerem orientação e treinamento específico. Darbyshire (2003), por exemplo, verificou grande variabilidade no conhecimento e entendimento dos pais sobre a hospitalização de seus filhos, com relatos indicando que parcela significativa dos pais não sabiam, ou não tinham sido informados, sobre o que esperar do processo de internação. Assim, faz-se necessário informar os pais sobre a doença, suas características, manejo e cuidados necessários durante a internação (Dodgson e cols., 2000; Moore \& Beckwitt, 2006) e após a alta hospitalar (Hewitt-Taylor, 2005).

Forinder (2004), em um estudo com mais de 10 anos de duração, realizou duas séries de entrevistas com pais de crianças que foram submetidas a transplante de medula óssea. A primeira entrevista era realizada no intervalo entre quatro e oito anos após o procedimento e a segunda, quatro anos após a primeira entrevista. Nas entrevistas, os pais eram convidados a contar a história vivenciada e, em seguida, o autor identificava os temas abordados. A análise apontou mecanismos mais eficientes de enfrentamento entre os pais que utilizaram mais da razão do que da emoção e que demonstraram uma participação mais ativa diante do tratamento e suas dificuldades.

Segundo Burke e cols. (2001) o treinamento dos pais é essencial, uma vez que, tal como a criança, os pais são submetidos a situações de estresse, especialmente aqueles expostos a episódios recorrentes de internação de seus filhos. Os autores propõem a utilização do SPIN (Stress-Point Intervention by Nurses), uma intervenção psicossocial e educacional que focaliza as preocupações dos pais. A técnica está dividida em três momentos: (a) a identificação do foco de estresse da família na situação de internação das crianças; (b) o desenvolvimento de um plano de intervenção específico para resolução do foco identificado; e (c) acompanhamento sistemático para avaliação dos efeitos da intervenção. Os autores destacam que a partir da técnica do SPIN as famílias passaram a adotar mecanismos mais eficientes de enfrentamento de situações de estresse e demonstraram maior satisfação com seu próprio funcionamento.

Além de discutir as intervenções com o paciente, seu acompanhante e a equipe de saúde, fazse necessário apresentar e discutir as técnicas e procedimentos mais utilizados para executar a preparação psicológica.

\section{Procedimentos de Preparação Psicológica}

Os procedimentos de preparação psicológica podem ser classificados diferentemente em função do critério adotado pelo(s) respectivo(s) autor(es). Bragado Álvarez e Fernandéz (1997), por exemplo, destacam procedimentos de preparação com fins informativos, distrativos e de desenvolvimento de enfrentamento.

Procedimentos informativos ou de transmissão de informação são os mais adotados, sendo que a informação oral prevalece em relação à escrita, apesar do aumento do uso de manuais educativos verificados na última década (Ferreira, 2005). Os fatores que tornam a informação o elemento principal de um processo de preparação psicológica incluem o alto potencial redutor de ansiedade, a eliminação de dúvidas simples acerca do procedimento e seus efeitos e o baixo custo operacional. Apesar disso, pesquisas demonstram que programas de preparação 
psicológica baseados unicamente no fornecimento de informação se mostram menos eficazes do que aqueles que envolvem uma combinação com outros procedimentos, tais como técnicas de relaxamento, distração e simulação (Caminiti, Scoditti, Diodati, \& Passalacqua, 2005).

Os recursos em audiovisuais, desde material em transparências de retroprojeção até filmes editados, são procedimentos utilizados em menor escala devido ao alto custo. No entanto, o filme apresenta várias vantagens: (a) constitui um recurso mais atrativo para crianças e adolescentes; (b) possibilita a utilização de efeitos visuais e sonoros que enfatizem determinadas informações-chave; (c) é um meio rápido e padronizado de fornecer informação, reduzindo o tempo de preparação e maximizando os benefícios aos pacientes; e (d) pode ser implantado facilmente no contexto hospitalar. Contudo, o filme apresenta, também, desvantagens: (a) pode ser um método impessoal de fornecimento de informação; (b) profissionalmente, sua elaboração envolve altos custos; e (c) os estímulos podem limitarse ao visual e ao auditivo (Quiles \& Carrillo, 2000).

Considerando a realidade de grande parte dos hospitais públicos brasileiros, os filmes poderiam constituir recursos didáticos potencialmente eficientes para a construção de repertórios de comportamentos colaborativos com o tratamento. Contudo, há que se considerar que a variedade de culturas e subculturas atendidas torna a elaboração deste material um desafio, além da falta de recursos financeiros e baixa disponibilidade de técnicos especializados para tal empreendimento, freqüentemente observada em serviços públicos de saúde.

Procedimentos distrativos, tais como jogos e brincadeiras, têm objetivo de desviar a atenção da criança do procedimento invasivo para uma atividade prazerosa (Bragado Álvarez \& Fernandéz, 1997). Destaca-se que procedimentos informativos e distrativos podem ser combinados; ao associar um jogo ao fornecimento de informação, permite-se, à criança, a expressão de sentimentos e pensamentos da forma que lhe é própria, de modo que o profissional de saúde possa dar atenção aos aspectos percebidos como mais estressantes pela criança (Costa Junior, Coutinho \& Ferreira, 2006).
Recentemente, devido ao avanço tecnológico, jogos eletrônicos têm sido muito utilizados como procedimentos distrativos. Patel e cols. (2006) investigaram a eficácia do videogame como técnica de distração e redução de ansiedade em períodos de pós-operatório pediátrico. Para tanto, os autores utilizaram videogames de mão que disponibilizavam uma variedade de 10 jogos cada um. Concluíram que o método é efetivo por constituir uma atividade instigante e prazerosa, que provê o alívio da ansiedade, provavelmente pela absorção cognitiva e motora da criança enquanto participa ativamente do jogo.

A utilização de jogos eletrônicos não se restringe à distração, podendo envolver também preparação ou treino para atividades específicas. Franck e Jones (2003), por exemplo, partindo do pressuposto de que ensinar técnicas cognitivas de manejo comportamental poderia reduzir a percepção de dor em crianças e os comportamentos indicadores de estresse durante a exposição a procedimentos médicos invasivos, desenvolveram um jogo de computador a partir do qual ensinam habilidades de enfrentamento para crianças em situação de espera para punção venosa. Neste jogo (CTCT - ComputerTaught Coping Techniques), são oferecidas seis opções de estratégias de enfrentamento à criança: (a) transmissão de informação; (b) técnicas de controle de respiração; (c) técnicas de relaxamento; (d) técnicas de distração; (e) técnicas de narração de procedimento com agulha; e (f) liberação de prêmios contingentes ao comportamento. Os principais resultados indicam a eficácia do software como redutor de ansiedade e grande aceitabilidade por parte de pais e profissionais de saúde.

Por fim, procedimentos de desenvolvimento de enfrentamento ensinam técnicas para a diminuição da atividade vegetativa excessiva (relaxamento, imaginação ativa, controle respiratório), técnicas de reestruturação cognitiva (auto-instruções e autoverbalizações), técnicas de promoção de condutas colaborativas (reforçamento diferencial, sistema de economia de fichas), entre outras. Estes procedimentos têm possibilitado o aumento da sensação de controle, diminuição das interpretações aterrorizantes sobre a situação e incremento da probabilidade de obter reforçamento positivo para comportamentos 
considerados adequados ao enfrentamento de situações estressantes (Bragado Alvarez \& Fernandéz, 1997; Quiles \& Carrillo, 2000; Roberts, 2003).

Anderson e Collier (1999), por exemplo, estudando a baixa adesão ao tratamento médico e seus potenciais efeitos colaterais em pacientes pediátricos, apresentaram um programa de intervenção no qual os pacientes eram admitidos na unidade, em seguida eram coletadas informações sobre a doença, o tratamento e a história da criança e da família e, então, executava-se um treinamento de habilidades para adesão que consistia de quatro etapas: (a) educação do paciente e da família quanto ao regime de tratamento; (b) treinamento de habilidades de auto-manejo identificadas pelo paciente e equipe como úteis à adesão; (c) intervenção comportamental utilizando contingências de reforçamento; e (d) tratamento das barreiras adicionais à adesão, tais como psicopatologias e dinâmicas familiares conflituosas. Para finalizar o programa, a família era treinada e preparada para a transferência dos cuidados para a casa, na medida em que o paciente respondia ao tratamento.

A partir desta breve revisão é possível sugerir alguns pontos-chave da preparação psicológica aplicada às crianças internadas:

(1) A intervenção deve ser realizada não apenas com a criança, mas também com seus pais (ou aqueles que desempenharem o papel de cuidadores primários);

(2) Deve ser desenvolvida, prioritariamente, nos momentos iniciais da internação, caracterizando-se seu caráter preventivo;

(3) Deve, na medida do possível, combinar diferentes métodos de intervenção (estratégias multifacetadas), com avaliações periódicas e sistemáticas de seus efeitos;

(4) Deve envolver, preferencialmente, toda a equipe multiprofissional e;

(5) Deve ser adaptada à realidade da unidade hospitalar e às necessidades dos usuários, a partir da avaliação psicossocial prévia;

Considerando esses pontos-chaves, apresentase, a seguir, a síntese de uma proposta de programa de preparação para admissão hospitalar infantil.

\section{Proposta de intervenção}

A proposta de intervenção, apresentada a seguir, foi estruturada em dois grupos de ações: preparatórias e práticas. $\mathrm{O}$ primeiro grupo refere-se às ações iniciais de elaboração e implantação de um programa de preparação para internação. Já o segundo, se refere à proposta, propriamente dita, de intervenção com os usuários (pacientes e familiares).

Cada grupo de ações foi subdividido e ordenado em passos e/ou etapas, que foram estabelecidos e ordenados a partir da experiência empírica, em curso, de implantação de um programa de preparação para admissão hospitalar infantil no Centro de Pediatria Clínica do HUB. A seguir, apresenta-se um quadro resumo dos dois grupos de ações:

\begin{tabular}{|c|c|c|}
\hline \multirow{6}{*}{$\begin{array}{c}\text { Ações } \\
\text { Preparatórias }\end{array}$} & $\begin{array}{c}1^{\circ} \\
\text { passo }\end{array}$ & $\begin{array}{l}\text { Envolvimento da equipe de } \\
\text { saúde }\end{array}$ \\
\hline & $\begin{array}{c}2^{\circ} \\
\text { passo }\end{array}$ & $\begin{array}{l}\text { Treino de habilidades sociais da } \\
\text { equipe de saúde }\end{array}$ \\
\hline & $\begin{array}{c}3^{\circ} \\
\text { passo }\end{array}$ & $\begin{array}{l}\text { Definição dos métodos e } \\
\text { delineamento do programa }\end{array}$ \\
\hline & $\begin{array}{c}4^{\circ} \\
\text { passo }\end{array}$ & $\begin{array}{c}\text { Organização dos recursos a } \\
\text { serem utilizados }\end{array}$ \\
\hline & $\begin{array}{c}5^{\circ} \\
\text { passo }\end{array}$ & $\begin{array}{l}\text { Treinamento da equipe e } \\
\text { divulgação do programa }\end{array}$ \\
\hline & $\begin{array}{c}6^{\circ} \\
\text { passo }\end{array}$ & $\begin{array}{l}\text { Execução e avaliação do } \\
\text { programa }\end{array}$ \\
\hline
\end{tabular}

\begin{tabular}{|c|c|c|}
\hline \multirow{1}{*}{ Ações Práticas } & $\begin{array}{c}1^{\mathrm{a}} \\
\text { etapa }\end{array}$ & $\begin{array}{c}\text { Recepção e acolhimento dos } \\
\text { usuários }\end{array}$ \\
\cline { 2 - 3 } & $\begin{array}{c}2^{\mathrm{a}} \\
\text { etapa }\end{array}$ & $\begin{array}{c}\text { Orientação e informação dos } \\
\text { usuários }\end{array}$ \\
\cline { 2 - 3 } & $\begin{array}{c}3^{\mathrm{a}} \\
\text { etapa }\end{array}$ & $\begin{array}{c}\text { Execução dos procedimentos de } \\
\text { rotina }\end{array}$ \\
\cline { 2 - 3 } & $\begin{array}{c}4^{\mathrm{a}} \\
\text { etapa }\end{array}$ & $\begin{array}{c}\text { Avaliações específicas das várias } \\
\text { especialidades }\end{array}$ \\
\cline { 2 - 3 } & $\begin{array}{c}5^{\mathrm{a}} \\
\text { etapa }\end{array}$ & $\begin{array}{c}\text { Execução dos programas de } \\
\text { preparação e acompanhamento }\end{array}$ \\
\cline { 2 - 3 } & $\begin{array}{c}6^{\mathrm{a}} \\
\text { etapa }\end{array}$ & Preparação para alta hospitalar \\
\hline
\end{tabular}

\section{Ações Preparatórias}

O primeiro passo para a implantação de um programa de preparação para a admissão hospitalar infantil consiste no envolvimento da equipe, de forma tal que todos sejam capazes de explicitar a importância 
desta intervenção e os benefícios que pode gerar tanto para crianças e pais, no que se refere à facilitação do processo de adaptação ao contexto de internação, quanto para a própria equipe profissional, ao: (a) reduzir as queixas típicas da internação; (b) prevenir comportamentos disfuncionais dos usuários, percebidos como fatores de estresse para os profissionais; e (c) reduzir os gastos hospitalares com a diminuição do tempo de internação, do uso de analgésicos e outros procedimentos técnicos (Barros, 1999; Costa Junior, 2004).

O segundo passo consiste no treinamento da equipe a fim de que a preparação para a internação seja uma intervenção qualificada e conjunta. Este treinamento deve englobar o desenvolvimento de habilidades sociais, tais como: escuta diferenciada, empatia, relacionamento profissional e social, comunicação simplificada, eficiência na resolução de problemas e prioridade para intervenção em crise. $\mathrm{O}$ desenvolvimento destas habilidades entre os profissionais de saúde constitui um preditor do estabelecimento de adequadas relações com os usuários, o que implica em taxas mais elevadas de adesão a tratamento (Koehn, 2006).

O terceiro passo consiste em definir, junto com a equipe, os métodos que serão utilizados no programa de preparação para a admissão hospitalar infantil, adequando-o à realidade filosófica, social e cultural do hospital e dos usuários atendidos. Este programa deve ser descrito e integrado aos documentos de normas e rotinas da unidade, dando-se ampla publicidade do mesmo entre os funcionários que atendem aos usuários do sistema de saúde.

$\mathrm{O}$ quarto passo consiste em providenciar os recursos necessários para a execução do programa de acordo com a metodologia definida na etapa anterior, ou seja, elaborar cartilha(s) informativa(s), gravar vídeo informativo/educativo, disponibilizar material lúdico relacionado a temáticas hospitalares com pessoal treinado para sua utilização, entre outros.

O quinto passo consiste na divulgação do programa e treinamento da equipe para executá-lo, com o objetivo de padronizar a conduta e eliminar dificuldades na sua execução.

O sexto e último passo consiste na execução do programa elaborado e na avaliação sistemática e periódica de seus efeitos a curto e médio prazo. Para tal, sugere-se que toda unidade pediátrica de internação mantenha uma equipe permanente de pesquisa em psicologia pediátrica.

\section{Ações Práticas}

Como de acordo com a proposta apresentada, o programa de preparação para internação deve ser um trabalho conjunto da equipe de saúde da unidade, a seguir, apresenta-se uma proposta de programa, baseada em dados colhidos a partir de um psicodiagnóstico institucional realizado no Centro Clínico do HUB (Doca, 2006).

A primeira etapa do programa consiste na recepção e acolhimento da criança e seu familiar na unidade. Este acolhimento envolve ações como chamar o paciente e seu acompanhante nominalmente, olhar nos olhos, realizar um breve rapport. $\mathrm{O}$ objetivo deste contato é iniciar uma relação de ajuda humana, na qual o profissional efetue uma escuta empática e livre, sendo capaz de acolher as expectativas do paciente e seu familiar, bem como avaliar os aspectos gerais de sua condição cognitiva e emocional.

A segunda etapa envolve a apresentação da unidade e o fornecimento de informações sobre as suas normas e rotinas de funcionamento, tais como horário de refeições, uso de vestimenta adequada e razões para tal, atividades desenvolvidas na unidade e rotinas médicas. Estas informações podem ser fornecidas oralmente, em linguagem simples e pessoal. Recomenda-se que, ao final das explicações, seja distribuído um folheto, didático e ilustrativo, contendo as principais informações, uma vez que a ansiedade inicial pode prejudicar a retenção de informação (Arora, 2003). Também sugere-se uma rápida verificação de entendimento das informações fornecidas, com a apresentação de poucas perguntas que devem ser respondidas pelo paciente(s) e acompanhante(s). Eventuais dificuldades de resposta indicam aspectos ou temáticas a serem revisadas ou abordadas oportunamente.

A terceira etapa consiste na realização dos procedimentos técnicos de rotina, conforme as normas de cada instituição hospitalar, que devem ser explicados antecipadamente (nomeados e descritos sucintamente) ao paciente(s) e acompanhante(s). 
A quarta etapa consiste na avaliação específica de cada especialidade. No caso da psicologia, crianças e familiares devem ser avaliados a fim de verificar as experiências e expectativas quanto à internação, nível de adesão ao tratamento e recursos de enfrentamento.

A quinta etapa consiste no estabelecimento de um programa de acompanhamento de acordo com as necessidades biopsicossociais dos usuários, avaliadas na ação anterior. O programa de acompanhamento psicológico pode envolver atendimentos individuais, técnicas de dessensibilização, relaxamento, treino de habilidades sociais, de enfrentamento, resolução de problemas, entre outros

Ao longo da internação, pode-se apresentar vídeos explicativos sobre temáticas hospitalares, que contenham dicas de comportamentos de enfrentamento tanto para o paciente quanto para o familiar, orientações sobre alimentação saudável, entre outros. Estes vídeos podem ser construídos com o apoio de pessoal especializado, utilizando-se a participação de crianças e familiares que já passaram pela experiência de hospitalização e/ou recursos de computação animada. Contudo, é importante salientar que nos casos de crianças submetidas à reinternação, a utilização do filme sobre procedimentos médicos ou rotinas hospitalares específicas deve ser avaliada, uma vez que pode ocorrer efeito de sensibilização, aumentando a ansiedade das crianças (Barros, 1999).

Um dos grandes diferenciais do programa de preparação para a internação consiste na postura dos profissionais diante das crianças e seus familiares. Além de afetiva e disponível, deve priorizar o atendimento às necessidades e direitos dos usuários, especialmente no que diz respeito à informação antecipada sobre todos os procedimentos a serem realizados e obtenção de consentimento informado.

É interessante realizar, semanalmente, reuniões interdisciplinares com os acompanhantes, envolvendo temas específicos e de interesse dos usuários. Essas reuniões podem ter o caráter tanto educativo (com palestras) quanto terapêutico (com espaço para exposição de sentimentos, sensações e cognições).

A última etapa do programa consiste na preparação para a alta hospitalar. Os pacientes e familiares devem ser orientados sobre como proceder em casa, a fim de dar continuidade ao tratamento e favorecer a superação das dificuldades que podem ser encontradas.

É importante estabelecer um processo avaliativo contínuo do programa, a partir do qual se possa identificar pontos deficitários e necessidades de ajustes, além de avaliar a eficácia do programa e o nível de satisfação dos usuários. Recomenda-se divulgar esses resultados no hospital e no meio científico, a fim de contribuir continuamente para o avanço do conhecimento da área.

Acredita-se que, com ações como estas, os efeitos adversos da internação podem ser reduzidos, e esta experiência contribua para a aquisição e manutenção de repertório comportamental funcional mais eficiente das crianças e seus familiares durante a admissão e a internação hospitalar.

\section{Referências}

Anderson, C. A., \& Collier, J. A. (1999). Managing very poor adherence to medication in children and adolescents: An inpatient intervention. Clinical Child Psychology and Psychiatry, 4, 393-402.

Araujo, T. C. C. F., \& Tubino, P. J. G. (1996). Efeitos da participação parental em rotina de centro cirúrgico para a adaptação psicológica do paciente pediátrico. Psicologia: Reflexão e Crítica, 9, 369-382.

Arora, N. K. (2003). Interacting with cancer patients: The significance of physicians' communication behavior. Social Science and Medicine, 57, 791806.

Barros, L. (1999). Psicologia pediátrica: Perspectiva desenvolvimentista. Lisboa: Climepsi.

Beck, A. T. (1976). Cognitive therapy and the emotional disorders. New York: International Universities Press.

Betz, C. L. (2006). Surgical preoperative preparation for children: The need for more evidence from nurse scientists. Journal of Pediatric Nursing, 21, 397-399. 
Blount, R. L., Cohen, L. L., Frank, N. C., Bachanas, P. J., Smith A. J., Manimala, M. R., \& Pate, J. T. (1997). The child-adult medical procedure interaction scale-revised: An assessment of validity. Journal of Pediatric Psychology, 22, 73-88.

Blount, R. L., Landolf-Fritsche, B., Powers, S. W., \& Sturges, J. W. (1991). Differences between high and low coping children and between parent and staff behaviors during painful medical procedures. Journal of Pediatric Psychology, 16, 795-809.

Blount, R. L., Piira, T., Cohen, L. L., \& Cheng, P. S. (2006). Pediatric procedural pain. Behavior Modification, 30, 24-49.

Bragado Álvarez, C., \& Fernandéz, A. M. (1997). Psychological treatment of evoked pain and anxiety by invasive medical procedures in paediatric oncology. Psychology in Spain, 1, 17-36.

Bronfenbrenner, U. (1979). The ecology of human development: Experiments by nature and design. Cambridge: Harvard University Press.

Burke, S. O., Harrison, M. B., Kauffmann, E., \& Wong, C. (2001). Effects of stress-point intervention with families of repeatedly hospitalized children. Journal of Family Nursing, 7, 128-158.

Caminiti, C., Scoditti, U., Diodati, F., \& Passalacqua, R. (2005). How to promote, improve and test adherence to scientific evidence in clinical practice [versão eletrônica]. BMC Health Services Research, 5, 62. Retirado em 25 janeiro 2006, de http://www.biomedcentral.com/1472-6963/5/62.

Cavender, K., Goff, M. D., Hollon, E. C., \& Guzzetta, C. E. (2004). Parent's positioning and distracting children during venipuncture: Effects on children's pain, fear, and distress. Journal of Holistic Nursing, 22, 32-56.

Chiattone, H. B. C. (2001). A família e a morte da criança. In V. A. Angerami-Camon (Org.), E a psicologia entrou no hospital (pp. 107-133). São Paulo: Pioneira \& Thomson Learning.

Corr, C. A. (1991). Support for grieving children: The Dougy Center and the hospice philosophy. The American Journal of Hospice \& Palliative Care, 8, 23-27.
Costa Junior, A. L. (2001). O desenvolvimento da psico-oncologia: implicações para a pesquisa e intervenção profissional em saúde. Psicologia: Ciência e Profissão, 21, 36-43.

Costa Junior, A. L. (2002). Psicologia aplicada à odontopediatria: uma introdução. Estudos e Pesquisas em Psicologia, 2, 67-77.

Costa Junior, A. L. (2004). A intervenção psicológica no contexto de procedimentos médicos invasivos em oncologia pediátrica: discussão metodológica. In M. Z. S. Brandão (Org.), Sobre comportamento e cognição (pp. 139-151). Santo André, SP: Esetec.

Costa Junior, A. L., Coutinho, S. M. G., \& Ferreira, R. S. (2006). Recreação planejada em sala de espera de uma unidade pediátrica: efeitos comportamentais. Paidéia (Ribeirão Preto), 16, 111-118.

Crawford, D. A. (2002). Keep the focus on the family. Journal of Child Health Care, 6, 133-146.

Crepaldi, M. A., Rabuske, M. M., \& Gabarra, L. M. (2006). Modalidades de atuação do psicólogo em psicologia pediátrica. In M. A. Crepaldi, M. B. M. Linhares \& G. B. Perosa (Orgs.), Temas em psicologia pediátrica (pp. 13-55). São Paulo: Casa do Psicólogo.

Darbyshire, P. (2003). Mother's experiences of their child's recovery in hospital and at home: A qualitative investigation. Journal of Child Health Care, 7, 291-312.

Doca, F.N.P. (2006). Psicodiagnóstico institucional da enfermaria do Centro de Clínicas Pediátricas do Hospital Universitário de Brasília. Manuscrito não publicado.

Dodgson, J. E., Garwick, A., Blozis, S. A., Patterson, J. M., Bennett, F. C., \& Blum, R. W. (2000). Uncertainty in childhood chronic conditions and family distress in families of young children. Journal of Family Nursing, 6, 252-266.

Ferreira, R. S. (2005). Efeitos da apresentação sistematizada de um manual educativo para pais de crianças com leucemia. Dissertação de mestrado não-publicada, Universidade de Brasília, Brasília, DF. 
Forinder, U. (2004). Bone marrow transplantation from a parental perspective. Journal of Child Health Care, 8, 134-148.

Franck, L., \& Jones, M. (2003). Computer-taught coping techniques for venepuncture: Preliminary findings from usability testing with children, parents and staff. Journal of Child Health Care, 7 , 41-54.

Guimarães, S. S. (1988). A hospitalização na infância. Psicologia: Teoria e Pesquisa, 4, 102-112.

Guimarães, S. S. (1999). Psicologia da saúde e doenças crônicas. In R. R. Kerbauy (Org.), Comportamento e saúde: Explorando alternativas (pp. 22-45). Santo André, SP: Arbytes.

Hewitt-Taylor, J. (2005). Caring for children with complex needs: Staff education and training. Journal of Child Health Care, 9, 72-86.

Junqueira, M. F. P. S. (2003). A mãe, seu filho hospitalizado e o brincar: um relato de experiência. Estudos de Psicologia (Natal), 8, 193-197.

Koehn, P. (2006). Globalization, migration health, and educational preparation for transnational medical encounters [versão eletrônica]. Globalization and Health, 2(2). Retirado em 10 fevereiro 2006, de http://www.globalizationandhealth.com/ content $/ 2 / 1 / 2$.

Lazarus, R. S., \& Folkman, S. (1984). Stress, appraisal, and coping. New York: Springer.

Martins, M. R., Ribeiro, C. A., Borba, R. I. H., \& Silva, C. V. (2001). Protocolo de preparo da criança pré-escolar para punção venosa, com utilização do brinquedo terapêutico. Revista Latino-Americana de Enfermagem, 9, 76-85.

Moore, J. B., \& Beckwitt, A. E. (2006). Self-care operations and nursing interventions for children with cancer and their parents. Nursing Science Quarterly, 19, 147-156.

Ogden, J. (1999). Psicologia da saúde. Lisboa: Climepsi.

Oliveira, H. (1993). A enfermidade sob o olhar da criança hospitalizada. Cadernos de Saúde Pública, 9, 326-332.
Oliveira, H. (1997). Ouvindo a criança sobre a enfermidade e a hospitalização. In R. B. Ceccim \& P. R. A. Carvalho (Orgs.), Criança hospitalizada: Atenção integral como escuta à vida (pp. 42-55). Porto Alegre: UFRGS.

Patel, A. D., Schieble, T., Davidson, M., Tran, M. C. J., Shoenberg, C., Delphin, E., \& Bennett, H. (2006). Distraction with a hand-held video game reduces pediatric preoperative anxiety. Pediatric Anesthesia, 16, 1019-1027.

Quiles, J. M. O., \& Carrillo, F. X. M. (2000). Hospitalización infantil. Repercusiones psicológicas. Teoria y práctica. Madrid: Biblioteca Nueva.

Ramsay, M. A. E. (2000). Acute postoperative pain management. Baylor University Medical Center Proceedings, 13, 244-247.

Reis, J. C. (1998). O sorriso de Hipócrates: A integração biopsicossocial dos processos de saúde e doença. Lisboa: Vega.

Roberts, M. C. (2003). Handbook of pediatric psychology (3rd ed.). New York: Guilford Press.

Scott, L. D., \& Arslanian-Engoren, C. (2005). The decision to care: A life-altering experience. Home Health Care Management \& Practice, 17, 130-135.

Soares, M. R. Z., \& Bomtempo, E. (2004). A criança hospitalizada: Análise de um programa de atividades preparatórias para procedimento médico de inalação. Estudos de Psicologia (Campinas), 21, 53-64.

Wade, G. H. (1999). Using the case method to develop critical thinking skills for the care of high-risk families. Journal of Family Nursing, 5, 92-109.

Willemsen, H., Chowdhury, U., \& Briscall, L. (2002). Needle phobia in children: A discussion of aetiology and treatment options. Clinical Child Psychology and Psychiatry, 7, 609-619.

World Health Organization. Division of Mental Health. (1993). Preparation for invasive procedures. Geneva: World Health Organization. 
Zannon, C. M. L. C. (1981). Atuação do psicólogo em setores de assistência pediátrica hospitalar. Boletim de Psicologia, 33, 40-51.

Artigo recebido em 12/03/2007.

Aceito para publicação em 05/07/2007.

Endereço para correspondencia:

Áderson Luiz Costa Junior. SQN 206 Bloco G, Apto 603, CEP: 70844-070. Brasília- DF, Brasil. Email: aderson@unb.br

Fernanda Nascimento Pereira Doca é Psicóloga do Hospital Universitário de Brasília. Especialista em Psicologia Hospitalar e da Saúde. Mestranda do Programa de Pós-graduação em Processos de Desenvolvimento Humano e Saúde, Universidade de Brasília.

Áderson Luiz Costa Junior é Professor Adjunto do Instituto de Psicologia da Universidade de Brasília. 